\title{
Chaotic Pulse Generation Induced by a Specific Class of Autonomous Oscillator
}

\author{
Ndombou GB${ }^{1}$, Marquié $P^{2}$, Fomethe $A^{3}$, Yemélé $D^{3}$, Jeutho $M G^{3}$ and Kenmogne $F^{4 *}$ \\ ${ }^{1}$ Laboratory of Electronics and Signal Processing, Faculty of Science, University of Dschang, Dschang, Cameroon \\ ${ }^{2}$ Laboratory of Electronic, Informatics and Image (LE2I), University of Burgundy, Dijon Cedex, France \\ ${ }^{3}$ Laboratoire de Mécanique et de Modélisation des Systèmes Physiques L2MSP, Faculté des Sciences, Université de Dschang, Cameroon \\ ${ }^{4}$ Laboratory of Modelling and Simulation in Engineering, Biomimetics and Prototype, Faculty of Science, University of Yaoundé I, Yaoundé, Cameroon
}

\begin{abstract}
The nonlinear dynamics of an autonomous chaotic oscillator, using two different stages operational amplifier coupled by mean of diode employed as the nonlinear device, recently introduced by Giannakopoulos and Deliyannis is considered with some particular modifications. These modifications are necessary for generating new type of oscillations, the regular and chaotic pulse oscillations according to the nature of operational amplifiers. Based on the nonlinear diode equation, the transfer voltage function of operational amplifiers in open loop configuration, and an appropriate selection of the state variables, a mathematical model is derived for a better description of the dynamics of the system. The complexness of oscillations is characterized using the bifurcation diagrams and the phase portraits. Some PSPICE simulations of the nonlinear dynamics of the oscillator are presented in order to confirm the ability of the oscillator to generate both the regular and chaotic pulse oscillations, according to the appropriate choice of its components.
\end{abstract}

Keywords: Pulse signals; Chaotic pulse signals; Transient chaotic pulse signal; Autonomous oscillator

\section{Introduction}

In recent years, communications via open networks such as satellites and internet occur more and more frequently. In most communication society, the transmitted messages are not to the public destination and must be protected against the pirate's access. In digital communications, only periodic pulse are used as carrier wave to modulate signals before their transmission [1], which remain a very promising practical application since the modulated signal by periodic pulse will propagate over very long distance without a significant attenuation, but remains nevertheless unsatisfactory since these information can easily be accessible.

Recent works on chaotic communication have revealed that information secured by chaos can be transferred from one place to another with higher security [2,3]. Since the chaotic signal is nonperiodic, it cannot be stored in the receiver as a reference in order to achieve coherent detection of the transmitted signal [4]. This is why the interest in chaotic oscillators [5-7] and their possible applications in secure communication remain increasing. The use of fully digital chaos communication, necessary for the confidentiality of information requires a chaotic pulse train, which is not easy to generate [1]. The chaotic pulse train means the inter-spike interval has a broad distribution and is uncorrelated. Chaotic pulses have been already found in the literature $[1,8]$ but the circuits are not simple and the pulse generation need a given chaotic time series and a pulse converter, using for a deep amplitude modulation of stationary chaotic signal at the output of chaotic source and which require permanent operation of chaotic oscillator. Chaotic radio-pulses are used as an information carrier in wideband and ultra-wideband communication systems $[9,10]$.

More recently [11], the autonomous chaotic oscillator, consisting of the Deliyannis single amplifier biquad $[11,12]$ and a LC resonant circuit coupled by means of a diode has been considered. In this chaotic oscillator, the negative resistance has been introduced in order to preserve the oscillations and has been implemented by using a negative impedance converter. It has been proved that this oscillator is described by a set of four differential equations, which exhibits a chaotic-like behavior, according to the resistance of the negative resistor.

In this paper, we reconsider the chaotic oscillator previously introduced in [11], in which some particular modifications have been carried out in order to introduce new effects on its dynamics, and we study the effects due to operational amplifiers on the dynamics of the system. To this end, the paper is organized as follows: In Section 2 we present the circuit under consideration, derive the equation of state and study the fixed point stability. In section 2, Numerical simulations will be carried out first, based on the derived state equations, in order to study the dynamic bifurcation and the pulse train generation. Next Pspice simulations are used to check numerical investigations and to justify the ability of the system to generate chaotic pulse train-like signal. Finally concluding remarks are devoted to section 3 .

\section{Circuit Description and State Equation}

\section{Circuit description}

In this section, we describe the physical structure of the autonomous chaotic oscillator as depicted in Figure 1. This oscillator consists of two different stages operational amplifiers connected by means of a nonlinear diode. The first stage contains the linear inductor $L$, the linear capacitor, the resistors $R_{0}, R_{1}$ and $R_{2}$ in the positive feedback, and also the resistors $R_{0}$ and $R_{3}$ in the negative feedback. In this stage, the operational amplifier associate to both two identical resistors, $R_{0}$ and $R_{3}$ act as negative resistance (if the voltages at the positive and negative

*Corresponding author: Kenmogne F, Laboratory of Modelling and Simulation in Engineering, Biomimetics and Prototype, Faculty of Science, University of Yaoundé I, Po Box 812, Yaoundé, Cameroon, Tel: +237 222213 20; E-mail: kenfabien@yahoo.fr

Received March 09, 2016; Accepted March 15, 2016; Published April 10, 2016

Citation: Ndombou GB, Marquié P, Fomethe A, Yemélé D, Jeutho MG, et al. (2016) Chaotic Pulse Generation Induced by a Specific Class of Autonomous Oscillator. J Electr Electron Syst 5: 181. doi:10.4172/2332-0796.1000181

Copyright: (c) 2016 Ndombou GB, et al. This is an open-access article distributed under the terms of the Creative Commons Attribution License, which permits unrestricted use, distribution, and reproduction in any medium, provided the original author and source are credited. 

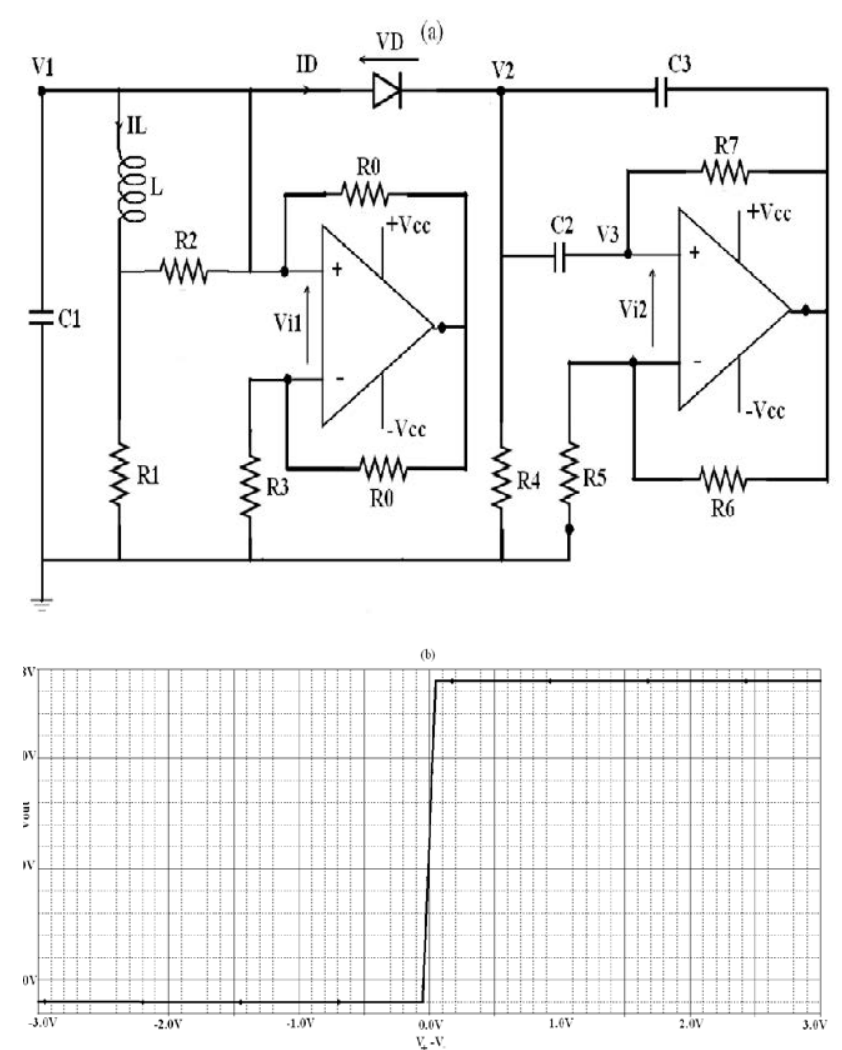

Figure 1: (a) Schematic diagram of the chaotic pulse oscillator. (b) Pspice transfer voltage characteristic, vout vs vin $=(v+-v-)$ of the operational amplifier in open loop configuration, showing its tan $\mathrm{h}$ model.

terminals are supposed to be identical) and can reduce to a negative resistance which $-R_{3}$ is introduced to preserve oscillations. The second stage contains both the linear capacitors $c_{2}$ and $c_{3}$, the resistors $R_{4}$ and $R_{7}$ in the positive feedback, and also the resistors $R_{5}$ and $R_{6}$ in the negative feedback. We notice that in [11], the resistors $R_{1}$ and $R_{2}$ had been not introduced and the effects due to operational amplifiers were neglected. In present work and in order to approximate our studies to the results which can be obtained with physical realistic operational amplifiers with the best accuracy, we suppose that the voltage difference between the positive and negative terminals $V_{\text {in }}=V_{+}-V$ operational amplifiers are nonzero, that is the operational amplifiers are supposed to be non ideal. As proved in Figure 1a, the transfer voltage function characteristic of the operational amplifier in the open loop configuration, from input to output is nonlinear and is expressed as

$$
v_{\text {out }}=V_{c c} \tanh \left(\beta \frac{v_{\text {in }}}{V_{c c}}\right) \text {, }
$$

Where $\beta$ is the voltage gain of operational amplifiers and the supply voltage used.

\section{Equation of state}

Denoting by $i_{\mathrm{L}}$ the current flowing through the inductor L, $v_{1}$ the voltage across the capacitor $c_{1}, v_{2}$ and $v_{3}$ he voltages at the left and the right of the capacitor $c_{1}$, respectively, and applying Kirchhoff's laws to the circuit of Figure 1, we obtain the following set of ordinary differential equations governing the dynamics of the system:

$$
c_{1} \frac{d v_{1}}{d t}=-\left(\frac{1}{R_{1}+R_{2}}-\frac{1}{R_{3}}\right) v_{1}-\left(\frac{1}{R_{0}}+\frac{1}{R_{3}}\right) v_{i 1}-\frac{R_{2}}{R_{1}+R_{2}} i_{L}-i_{d},
$$

$$
\begin{aligned}
& L \frac{d i_{L}}{d t}=\frac{R_{2}}{R_{1}+R_{2}}\left(v_{1}-R_{1} i_{L}\right), \\
& c_{3} \frac{d v_{2}}{d t}=\frac{1}{1-\left(1+\frac{R_{5}}{R_{6}}\right) \frac{d v_{i 2}}{d v_{3}}}\left[\frac{R_{5}}{R_{6}}\left(\frac{v_{2}}{R_{4}}-i_{d}\right)+\frac{1}{R_{7}}\right. \\
& \left(\left(1+\frac{R_{5}}{R_{6}}\right) v_{i 2}-v_{3}\right)\left[1+\frac{c_{3}}{c_{2}}\left(1+\frac{R_{6}}{R_{5}}\left(1-\left(1+\frac{R_{5}}{R_{6}}\right) \frac{d v_{i 2}}{d v_{3}}\right)\right)\right],
\end{aligned}
$$

where $i_{d}$ is the current in the diode, which is related to the diode voltage as

$$
\begin{aligned}
& \mathrm{V}_{\mathrm{d}}=\mathrm{V}_{1}-\mathrm{V}_{2} \\
& i_{d}=I_{s}\left(\exp \left(\frac{v_{d}}{\eta V_{T}}\right)-1\right),
\end{aligned}
$$

where $I_{S}$ is the saturation current of the junction, $V_{t}$ is the thermal voltage [13]. This thermal voltage is proportiona 1 to absolute temperature and takes the value $26 \mathrm{mV}$ at room temperature, that is at $293 \mathrm{~K}$, while $\eta$, with $1<\eta<2$ is the ideality factor of the diode. The input voltages $\mathrm{v}_{\mathrm{i} 1}$ and $\mathrm{v}_{\mathrm{i} 2}$, are related to $\mathrm{v}_{1}$ and $\mathrm{v}_{2}$ by the following equations:

$$
\begin{aligned}
& \mathrm{v}_{1}=\mathrm{v}_{\mathrm{i} 1}+\frac{\mathrm{R}_{3} \mathrm{v}_{\mathrm{cc}}}{\mathrm{R}_{0}+\mathrm{R}_{3}} \tanh \left(\frac{\beta \mathrm{v}_{\mathrm{i} 1}}{\mathrm{v}_{\mathrm{cc}}}\right), \\
& \mathrm{v}_{3}=\mathrm{v}_{\mathrm{i} 2}+\frac{\mathrm{R}_{5} \mathrm{v}_{\mathrm{cc}}}{\mathrm{R}_{5}+\mathrm{R}_{6}} \tanh \left(\frac{\beta \mathrm{v}_{\mathrm{i} 1}}{\mathrm{v}_{\mathrm{cc}}}\right),
\end{aligned}
$$

while $\mathrm{dv}_{\mathrm{i} 1} / \mathrm{dv}_{2}$ is the derivative of $\mathrm{v}_{\mathrm{i} 2}$ in term of $\mathrm{v}_{3}$ When the operational amplifiers are supposed to be ideal, that is when $v_{i 1}=v_{i 2}=$ 0 , the set of Eq.(2) reduces to the following set of differential equations:

$$
\begin{aligned}
& \mathrm{c}_{1} \frac{\mathrm{dv}_{1}}{\mathrm{dt}}=-\left(\frac{1}{\mathrm{R}_{1}+\mathrm{R}_{2}}-\frac{1}{\mathrm{R}_{3}}\right) \mathrm{v}_{1}-\frac{\mathrm{R}_{2}}{\mathrm{R}_{1}+\mathrm{R}_{2}} \mathrm{i}_{\mathrm{L}}-\mathrm{i}_{\mathrm{d}}, \\
& \mathrm{L} \frac{\mathrm{di}_{\mathrm{L}}}{\mathrm{dt}}=\frac{\mathrm{R}_{2}}{\mathrm{R}_{1}+\mathrm{R}_{2}}\left(\mathrm{v}_{1}-\mathrm{R}_{1} \mathrm{i}_{\mathrm{L}}\right), \\
& \mathrm{c}_{3} \frac{\mathrm{dv}}{\mathrm{dt}}=\frac{\mathrm{R}_{5}}{\mathrm{R}_{6}}\left(\frac{\mathrm{v}_{2}}{\mathrm{R}_{4}}-\mathrm{i}_{\mathrm{d}}\right)-\frac{\mathrm{v}_{3}}{\mathrm{R}_{7}}\left(1+\frac{\mathrm{c}_{3}}{\mathrm{c}_{2}}\left(1+\frac{\mathrm{R}_{6}}{\mathrm{R}_{5}}\right)\right), \\
& \mathrm{c}_{3} \frac{\mathrm{dv}}{\mathrm{dt}}=\frac{\mathrm{R}_{5}}{\mathrm{R}_{6}}\left(\frac{\mathrm{v}_{2}}{\mathrm{R}_{4}}-\mathrm{i}_{\mathrm{d}}\right)-\frac{\mathrm{v}_{3}}{\mathrm{R}_{7}}\left(1+\frac{\mathrm{c}_{3}}{\mathrm{c}_{2}}\right),
\end{aligned}
$$

In order to modify the above set of differential equations in the dimensionless form in a way convenient for analytical and numerical analysis, we introduce the following change of variables:

$$
\begin{aligned}
& x_{1}=v_{1} / \eta V_{T}, x_{2}=\rho i_{L} / \eta V_{T}, x_{3}=v_{2} / \eta V_{T}, \\
& x_{4}=v_{3} / \eta V_{T}, y_{1}=v_{i 1} / \eta V_{T}, y_{2}=v_{i 2} / \eta V_{T}, \\
& t=\tau \sqrt{L c_{1}} \rho=\sqrt{L / c_{1}} .
\end{aligned}
$$

which lead the set of Eq. (2) to the following set of four ordinary differential equations governing signal voltage in the system

$$
\left\{\begin{array}{l}
\dot{x}_{1}=\sigma\left(x_{1}-y_{1}\right)-a_{1} y_{1}-\frac{1}{1+a_{0} b_{0}}\left(x_{2}+a_{0} x_{1}\right)-\gamma g\left(x_{1}, x_{3}\right), \\
\dot{x}_{2}=\frac{1}{1+a_{0} b_{0}}\left(x_{1}-b_{0} x_{2}\right), \\
\dot{x}_{3}=\frac{1}{1-(\varepsilon+1) \frac{d y_{2}}{d x_{4}}}\left[\varepsilon_{1} \varepsilon\left(b_{1} x_{3}-\gamma g\left(x_{1}, x_{3}\right)\right)+b_{2}\left(\varepsilon_{1}+\varepsilon_{2}\left(1+\frac{1}{\varepsilon}\right)\left(1-\frac{d y_{2}}{d x_{4}}\right)\right)\left((\varepsilon+1) y_{2}-x_{4}\right)\right], \\
\dot{x}_{4}=\frac{1}{1-(\varepsilon+1) \frac{d y_{2}}{d x_{4}}}\left[\varepsilon_{1} \varepsilon\left(b_{1} x_{3}-\gamma g\left(x_{1}, x_{3}\right)\right)+b_{2}\left(\varepsilon_{1}+\varepsilon_{2}\right)\left((\varepsilon+1) y_{2}-x_{4}\right)\right]
\end{array}\right.
$$


Citation: Ndombou GB, Marquié P, Fomethe A, Yemélé D, Jeutho MG, et al. (2016) Chaotic Pulse Generation Induced by a Specific Class of Autonomous Oscillator. J Electr Electron Syst 5: 181. doi:10.4172/2332-0796.1000181

with

$$
x_{1}=y_{1}+\frac{a_{1}}{\gamma_{1}\left(a_{1}+\sigma\right)} \tanh \left(\beta \gamma_{1} y_{1}\right), x_{4}=y_{2}+\frac{\varepsilon}{\gamma_{1}(1+\varepsilon)} \tanh \left(\beta \gamma_{1} y_{2}\right) \text {, }
$$

and

$$
\frac{d y_{2}}{d x_{4}}=\frac{\cosh ^{2}\left(\beta \gamma_{1} y_{2}\right)}{\frac{\varepsilon \beta}{(1+\varepsilon)}+\cosh ^{2}\left(\beta \gamma_{1} y_{2}\right)}, g\left(x_{1}, x_{3}\right)=\exp \left(x_{1}-x_{3}\right)-1 \text {. }
$$

where by the dots we mean the derivative with respect to $\tau$ and where the following parameters are introduced

$$
\begin{aligned}
& \sigma=\frac{\rho}{R_{3}}, a_{0}=\frac{\rho}{R_{2}}, a_{1}=\frac{\rho}{R_{0}}, b_{0}=\frac{R_{1}}{\rho}, b_{1}=\frac{\rho}{R_{4}}, b_{2}=\frac{\rho}{R_{7}}, \\
& \varepsilon=\frac{R_{5}}{R_{6}}, \varepsilon_{1}=\frac{c_{1}}{c_{3}}, \varepsilon_{2}=\frac{c_{1}}{c_{2}}, \gamma_{1}=\frac{\eta V_{T}}{v_{c c}}, \gamma=\frac{\rho I_{s}}{\eta V_{T}} .
\end{aligned}
$$

For simplifying our investigations, the following values of parameters have been kept constant as follows: $=4 \mathrm{k} \Omega$

$\mathrm{L}=188.8 \mathrm{mH}, \mathrm{R}_{1}=2 \mathrm{k} \Omega, \mathrm{R}_{2}=20 \Omega, \mathrm{R}_{0}=5.6 \mathrm{k} \Omega, \mathrm{R}_{4}=200 \mathrm{k} \Omega, \mathrm{R}_{5}$

$$
\mathrm{R}_{6}=100.1 \mathrm{k} \Omega, \mathrm{R}_{7}=12.99 \mathrm{k} \Omega, c_{1}=62 n F, c_{2}=60 n F, c_{3}=70 n F
$$

The nonlinear diode used is the D1N4148 model with the characteristics $I_{S}=2.682 \times 10^{-9} \mathrm{~A}$ and $\eta=1.9$ leading to the following parameters values:

$$
\begin{aligned}
& a_{0}=0.873, a_{1}=0.312, b_{0}=5307.045, \varepsilon=3.996 \times 10^{-2}, \\
& \varepsilon_{2}=\frac{31}{30}, \gamma=9.474 \times 10^{-5}, \gamma_{1}=3.293 \times 10^{-3}, \varepsilon_{1}=\frac{31}{35}, b_{1}=8.725 \times 10^{-3}, b_{2}=0.134
\end{aligned}
$$

while $\sigma$ (that is, $R_{3}$ since $\sigma=1745.039051 / R_{3}$ ) and $\beta$ are the tuning parameter

\section{Fixed point analysis}

It is easy to show that system [7] has one equilibrium point. $\mathrm{E}_{0}(0,0,0,0)$ Physically, a steady state solution corresponds to an equilibrium state of the system and the behavior of the system may depend on its stability. To test this stability, let us consider the state $\mathrm{E}=\mathrm{E}_{0}+\delta \mathrm{E}$ vector, where $\delta \mathrm{E}$ is the perturbation of the equilibrium solution $E_{0}(0,0,0,0)$. The stability of this equilibrium state against the perturbation $\delta E$ depends on the properties of the eigenvalues of the Jacobian matrix $J\left(E_{0}\right)$, which can be easily computed as follows:

$$
\left[\begin{array}{cccc}
-\left(\gamma+\frac{a_{0}}{1+a_{0} b_{0}}+\frac{a_{1}+\sigma(1-\beta)}{1+\beta+\sigma / a_{1}}\right) & -\frac{1}{1+a_{0} b_{0}} & \gamma & 0 \\
\frac{1}{1+a_{0} b_{0}} & -\frac{b_{0}}{1+a_{0} b_{0}} & 0 & 0 \\
\frac{\varepsilon_{1} \gamma(1+\varepsilon(1+\beta))}{1+\varepsilon-\beta} & 0 & \frac{\varepsilon_{1}\left(b_{1}+\gamma\right)(1+\varepsilon(1+\beta))}{\beta-1-\varepsilon} & -b_{2}\left(\varepsilon_{1}+\frac{\beta \varepsilon_{2}(1+\varepsilon)}{1+\beta(1+\varepsilon)}\right) \\
\frac{\varepsilon_{1} \gamma(1+\varepsilon(1+\beta))}{1+\varepsilon-\beta} & 0 & \frac{\varepsilon_{1}\left(b_{1}+\gamma\right)(1+\varepsilon(1+\beta))}{\beta-1-\varepsilon} & -b_{2}\left(\varepsilon_{1}+\varepsilon_{2}\right)
\end{array}\right]
$$

This Jacobean matrix evaluated at the equilibrium point $E_{0}$ satisfies the following characteristic equation:

$$
\lambda^{4}+j_{3} \lambda^{3}+j_{2} \lambda^{2}+j_{1} \lambda+j_{0}=0
$$

with

$$
j_{0}=\frac{\varepsilon_{1} \varepsilon_{2} b_{2}}{1+a_{0} b_{0}}\left[\left(\gamma+b_{1}\right)\left(1+b_{0}\left(\gamma+\frac{a_{1}+\sigma(1-\beta)}{1+\beta+\sigma / a_{1}}\right)\right)-\gamma^{2} b_{0}\right] \text {, }
$$

$$
\begin{aligned}
& j_{1}=\frac{j_{0}(1+\varepsilon(\beta+1))}{\varepsilon_{2} b_{2} \varepsilon(\beta-1-\varepsilon)}+b_{2}\left(\gamma+\frac{a_{0}}{1+a_{0} b_{0}}+\frac{a_{1}+\sigma(1-\beta)}{1+\beta+\sigma / a_{1}}\right) \\
& {\left[\varepsilon_{1} \varepsilon_{2}\left(\gamma+b_{1}\right)+\frac{\left(\varepsilon_{1}+\varepsilon_{2}\right) b_{0}}{1+a_{0} b_{0}}\right]+\frac{b_{2}\left(\varepsilon_{1}+\varepsilon_{2}\right)}{\left(1+a_{0} b_{0}\right)^{2}}+b_{0} b_{2} \varepsilon_{1} \varepsilon_{2}\left(\frac{\left(\gamma+b_{1}\right) b_{0}}{1+a_{0} b_{0}}-\gamma^{2}\right),}
\end{aligned}
$$
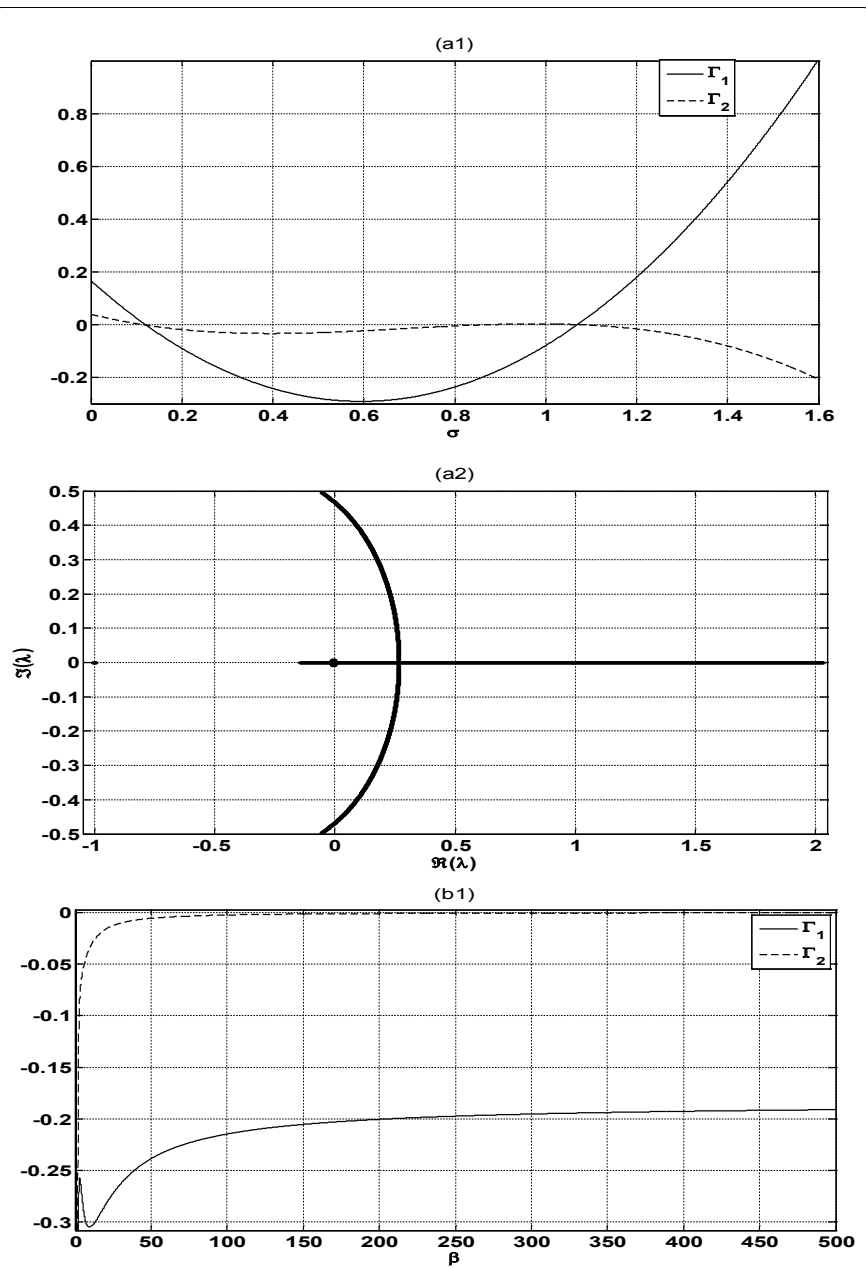

(b2)

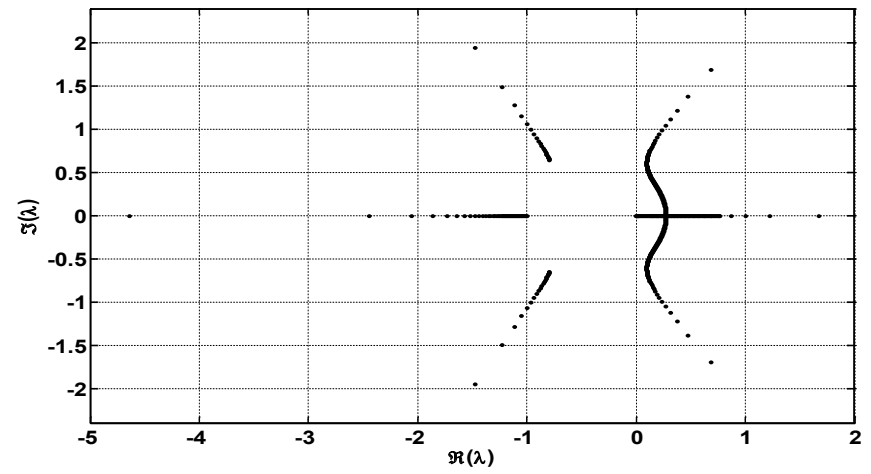

Figure 2: (a1) Parameters $\Gamma_{1}$ and $\Gamma_{2}$, and (a2) Representation in the complex plane of the eighteen values of the Jacobian matrix solutions of Eq. (14). The parameters of the system are listed in (12) (b1) $\beta=1500$ and the parameter $\sigma$ is used as the control parameter of the system. (b2) $\sigma=0.88$ and $\beta$ chosen as the control parameter. 
Citation: Ndombou GB, Marquié P, Fomethe A, Yemélé D, Jeutho MG, et al. (2016) Chaotic Pulse Generation Induced by a Specific Class of Autonomous Oscillator. J Electr Electron Syst 5: 181. doi:10.4172/2332-0796.1000181

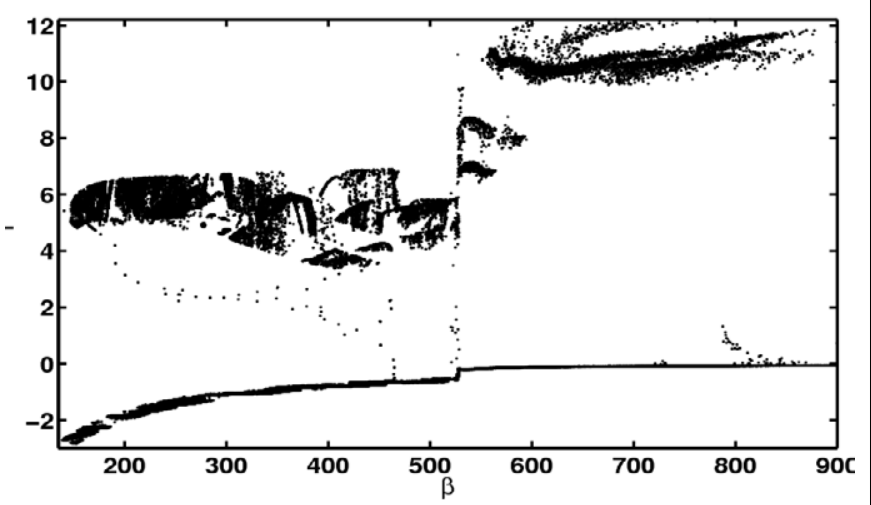

Figure 3: Bifurcation diagram obtained for $\sigma=0.88$ and the parameters given in Eq. 12, while is $\beta$ chosen as a control parameter.

(a)

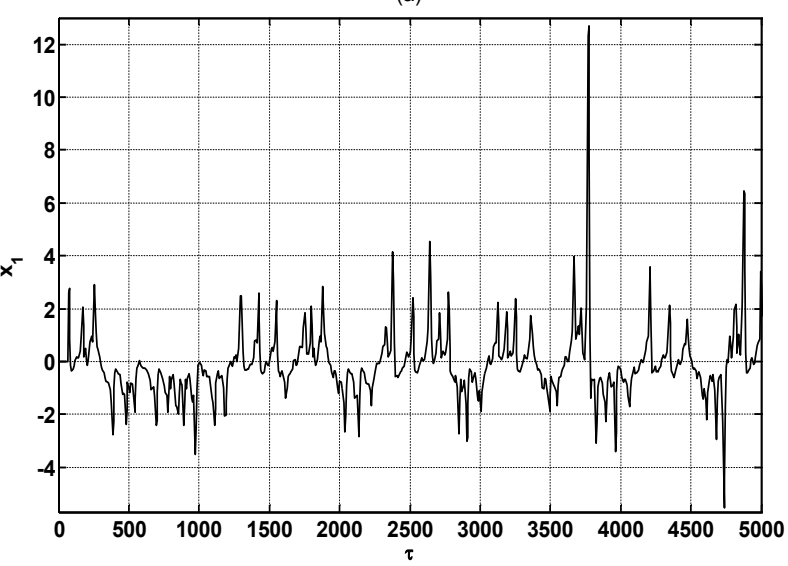

(b)

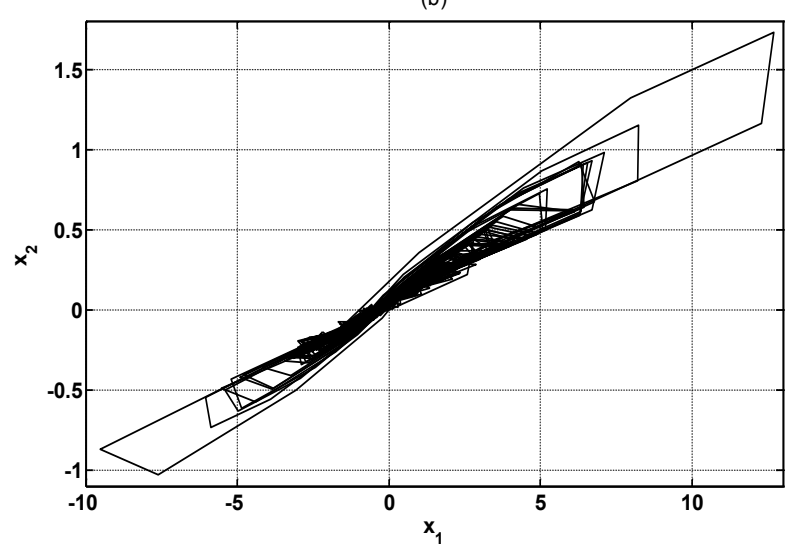

Figure 4: (a) Time waveform of variable $x_{1}(t)$, (b) Phase space plot $x_{2}$ against $x_{1}$. The parameters of the system are taken as in Figure 2, but with $\sigma=0.88$ and $\beta=1500$.

$$
\begin{aligned}
& j_{2}=\frac{\varepsilon_{1}(1+\varepsilon(\beta+1))}{1+\varepsilon-\beta}\left[\left(\gamma+b_{1}\right)\left(\gamma+\frac{b_{0}+a_{0}}{1+a_{0} b_{0}}+\frac{a_{-} 1+\sigma(1-\beta)}{1+\beta+\sigma / a_{1}}\right)-\gamma^{2}\right] \\
& +\left(\gamma+\frac{a_{0}}{1+a_{0} b_{0}}+\frac{a_{1}+\sigma(1-\beta)}{1+\beta+\frac{\sigma}{a_{1}}}\right)\left(\frac{b_{0}}{1+a_{0} b_{0}}+b_{2}\left(\varepsilon_{1}+\varepsilon_{2}\right)\right) b_{2}\left[\frac{b_{0}\left(\varepsilon_{1}+\varepsilon_{2}\right)}{1+a_{0} b_{0}}+\varepsilon_{1} \varepsilon_{2}\left(\gamma+b_{1}\right)\right],
\end{aligned}
$$

$$
j_{3}=\gamma+\frac{b_{0}+a_{0}}{1+a_{0} b_{0}}+\frac{a_{1}+\sigma(1-\beta)}{1+\beta+\sigma / a_{1}}-\frac{\varepsilon_{1}\left(\gamma+b_{1}\right)(1+\varepsilon(\beta+1))}{\beta-1-\varepsilon}+b_{2}\left(\varepsilon_{1}+\varepsilon_{2}\right)
$$

A set of necessary and sufficient conditions for all the roots of (14) to have negative real parts is given by the well-known Routh-Hurwitz criterion expressed in the form:

$$
\begin{aligned}
& j_{i}>0,(i=0,1,2,3), \quad \Gamma_{1}=j_{2} j_{3}-j_{1}>0, \\
& \Gamma_{2}=j_{3}\left(j_{1} j_{2}-j_{0} j_{3}\right)-j_{1}^{2}>0 .
\end{aligned}
$$

The plot of parameters $\Gamma_{1}$ and $\Gamma_{2}$ are shown in Figure 2 for certain choice of the system parameters, from where it appears that when $\beta$ $=1500$ and $\sigma$ chosen as a control parameter as shown in Figure 2a in one hand, the corresponding equilibrium set $\mathrm{E}_{0}$ is unstable for $\sigma$ $>\sigma_{0}=0.121$, which implies that the orbit of system [7] starting from the equilibrium set is unstable and asymptotically tend to limit cycle or attractor or infinite. Otherwise for $\sigma<\sigma_{0}$ the equilibrium set $\mathrm{E}_{0}$ is attractive, implying that regular oscillations may appear, but the system will not be chaotic. In the other hand when $\sigma=0.88$ and $\beta$ chosen as a control parameter as shown in Figure $2 b$, the equilibrium point $E_{0}$ is always unstable, provided that $\Gamma_{1}$ and $\Gamma_{2}$ are negatives.

(a)

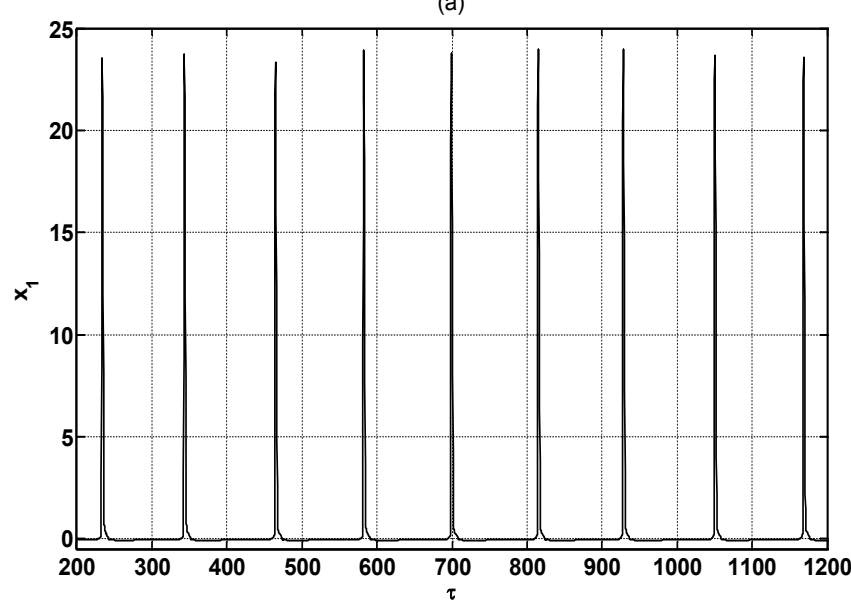

(b)

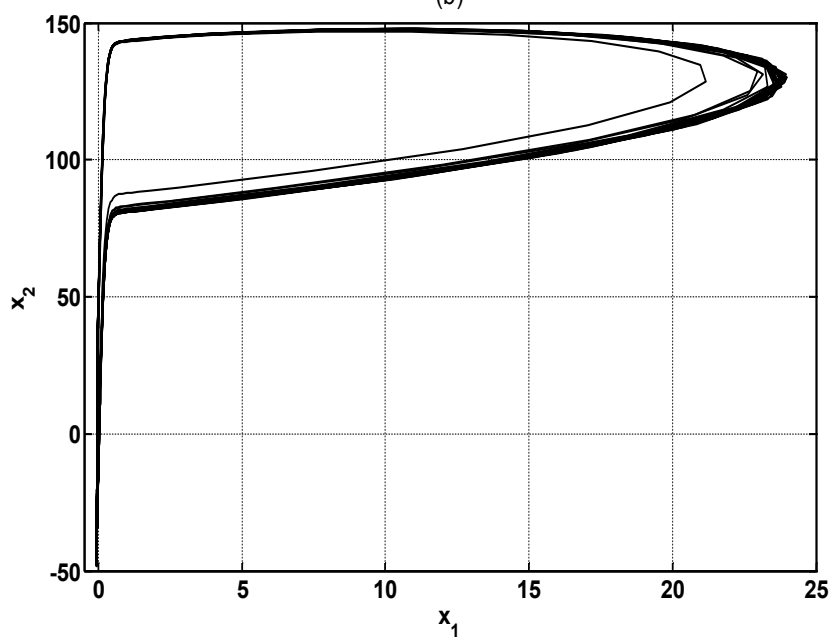

Figure 5: (a) Time waveform of $x_{1}(t)$ (b) Phase space plot $x_{2}$ against $x_{1}$. The parameters of the system are taken as in Figure 2, but with $\sigma=0.88$ and $\beta$ $=1500$. 
Citation: Ndombou GB, Marquié P, Fomethe A, Yemélé D, Jeutho MG, et al. (2016) Chaotic Pulse Generation Induced by a Specific Class of Autonomous Oscillator. J Electr Electron Syst 5: 181. doi:10.4172/2332-0796.1000181

Page 5 of 7

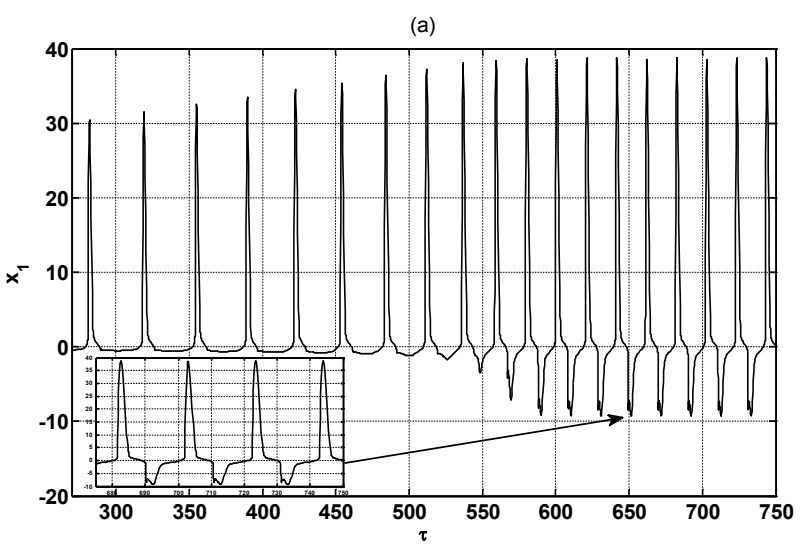

(b)

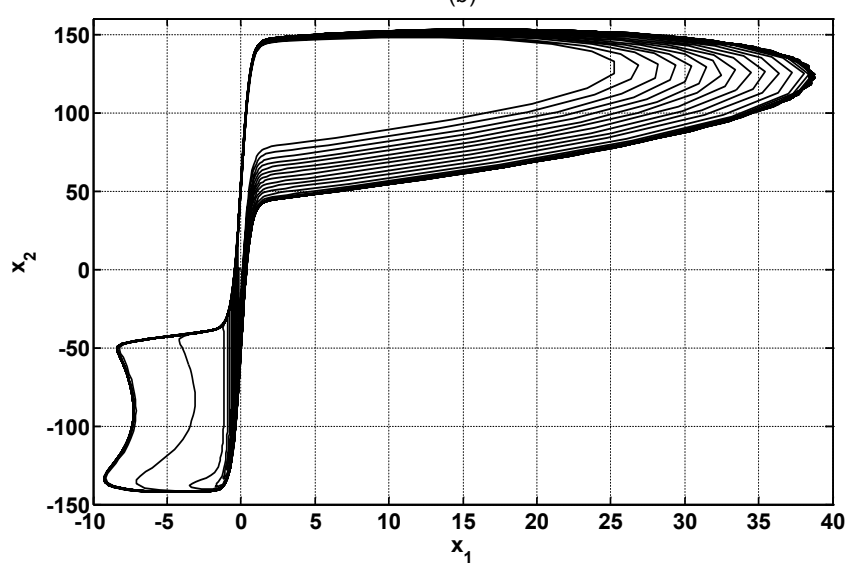

Figure 6: (a) Transient pulse like signal $x_{1}(t)$, (b) Phase space plot $x_{2}$ against $x_{1}$. The parameters of the system are taken as in Figure 2, but with $\sigma=0.88$ and $\beta=1500$.

\section{Results of Numerical and Pspice Simulations}

\section{Numerical investigations}

In order to investigate the dependency of the dynamics of the system to the choice of the operational amplifier, system [7-9] is integrated numerically using the classical fourth-order Runge-Kutta integration scheme, with the integration time step $\Delta \tau=0.002$ and the computations are performed out using variables and constants parameters in extended mode given in [12]. For $\sigma=0.88$, system [7-9] is integrated for a sufficiently long time to discard the transient, leading to the bifurcation diagram plotted as a function of the amplifier gain to prove the sensitivity of the system to the choice of the operational amplifier. This bifurcation diagram is obtained by plotting the local maxima of state variables in terms of the bifurcation control parameter $\beta$. As illustrated in Figure 3, when $\beta<140$, there is no oscillation in the system. Otherwise the dynamics of the system varies for increasing value of $\beta$ and rise to periodic 1 limit cycle for $\beta>875$. To confirm the ability of the system to generate regular and chaotic pulse-like signals, sample time traces with the corresponding phase portraits are computed for some discrete values of the control parameters $\sigma$ and $\beta$. The chaotic pulse train is obtained for $\beta=1500$ and $\sigma=0.98$ as depicted in Figure 4, while in Figure 5, only regular pulses are obtained. In Figure 6 it shows two aspects of the behavior of the system, the transient regular pulses, and the incoherent pulse train obtained after the transient.

\section{Pspice simulations and bifurcation of phase portrait}

Based on the theoretical analysis presented above, realistic Pspice simulations of the system shown in Figure 1 are simulated, in order to validate the mathematical model proposed in this work, and to evaluate the effects of ideal diode model and operational amplifiers on the dynamics of the system. For this end, the parameters used for the circuit simulation are chosen as indicated in Eq. (11), with varying values of $\mathrm{R}_{3}$, first in order to confirm the sensitivity of the system with respect to

(al)
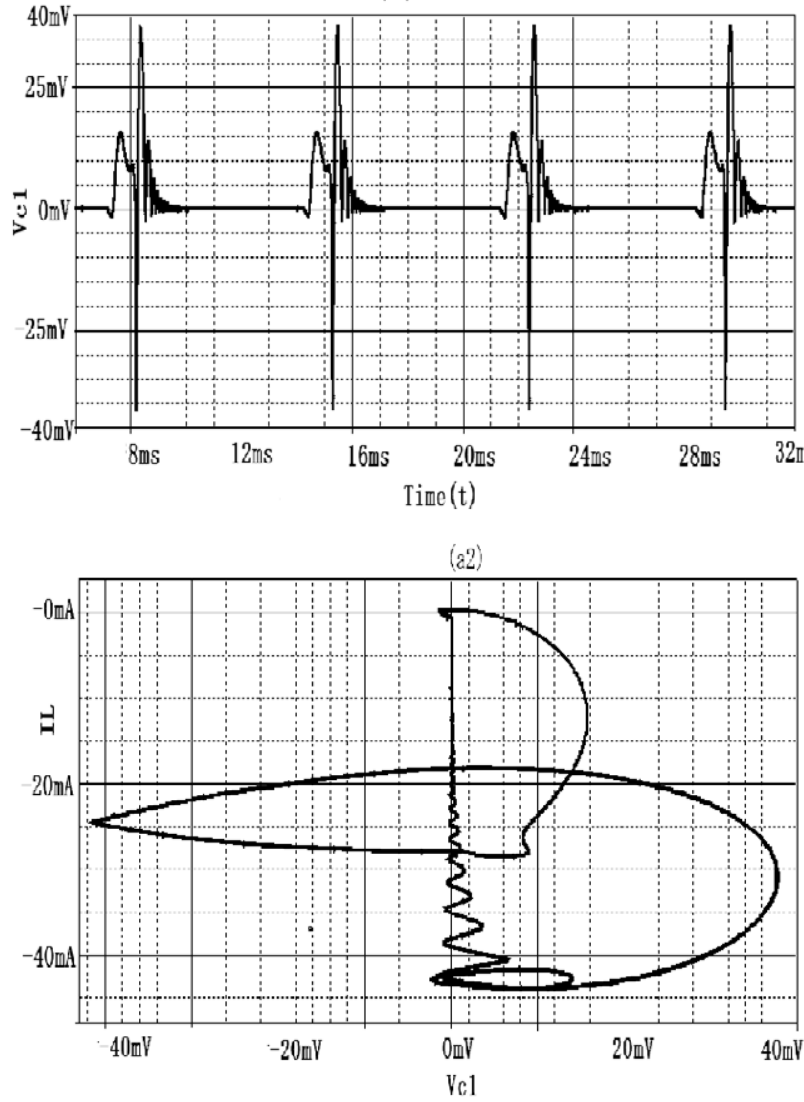

(b1)

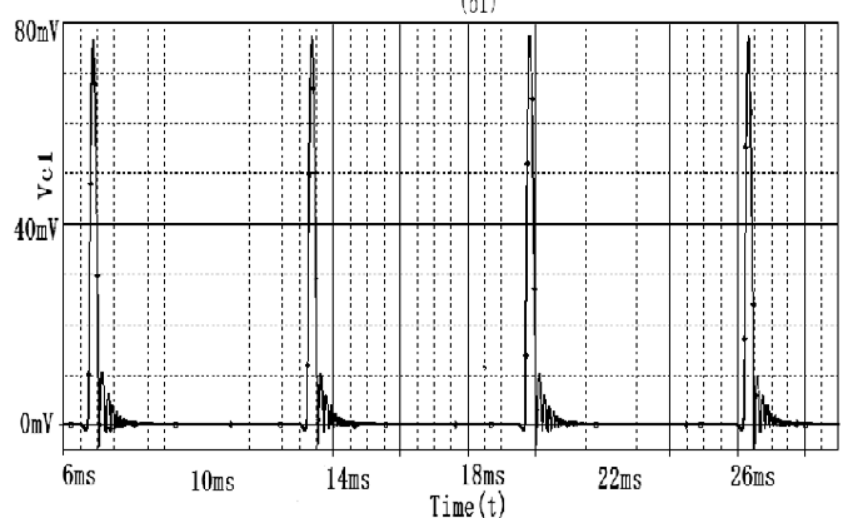

Figure 7: Results of the Pspice simulations, obtained for the LM759CP mode of operational amplifier, and for the values of parameters given in Eq (11). (a1) Time dependent signal voltage $v_{1}(t),(a 2)$ Phase space plot. (a) is obtained for $R_{3}=5 \mathrm{k} \Omega$, while (b1) is obtained for $R_{3}=10 \mathrm{k} \Omega$. As one can see, the system exhibits the periodic modulated pulse signals. 
Citation: Ndombou GB, Marquié P, Fomethe A, Yemélé D, Jeutho MG, et al. (2016) Chaotic Pulse Generation Induced by a Specific Class of Autonomous Oscillator. J Electr Electron Syst 5: 181. doi:10.4172/2332-0796.1000181

Page 6 of 7

(bl)

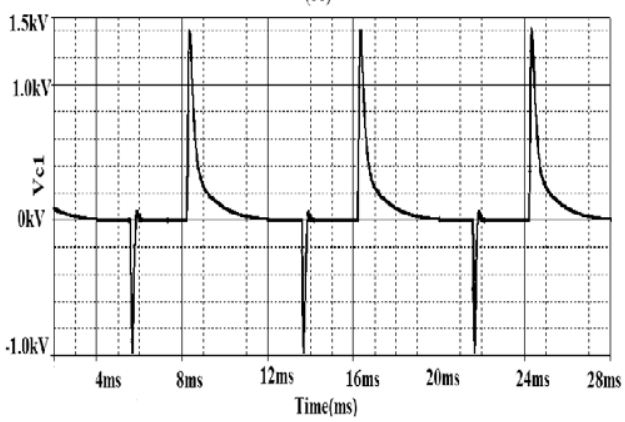

(b2)

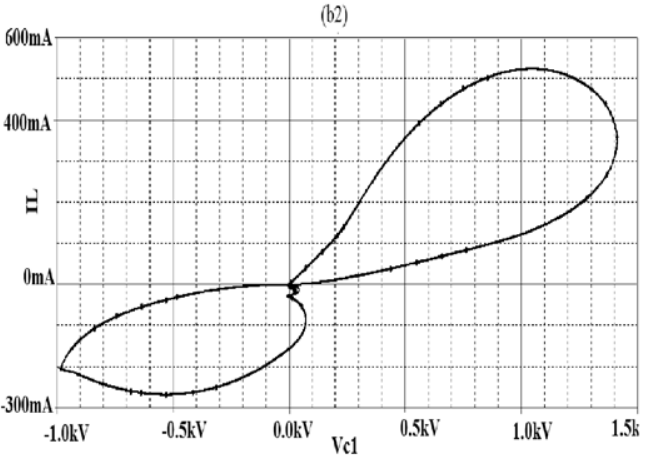

Figure 8: Results of the Pspice simulations, obtained for $R_{3}=5 \mathrm{k} \Omega$ and for the LM675 model of operational amplifier. The values of the others parameters are given in Eq.(11). (b1): Time dependent signal voltage $v_{1}(t)$, (b2): Phase space plot. The phase portrait is the homoclinic orbit, that is the orbit which begins on one fixed point and end on the same fixed point, corresponding to pulse train-like signals.
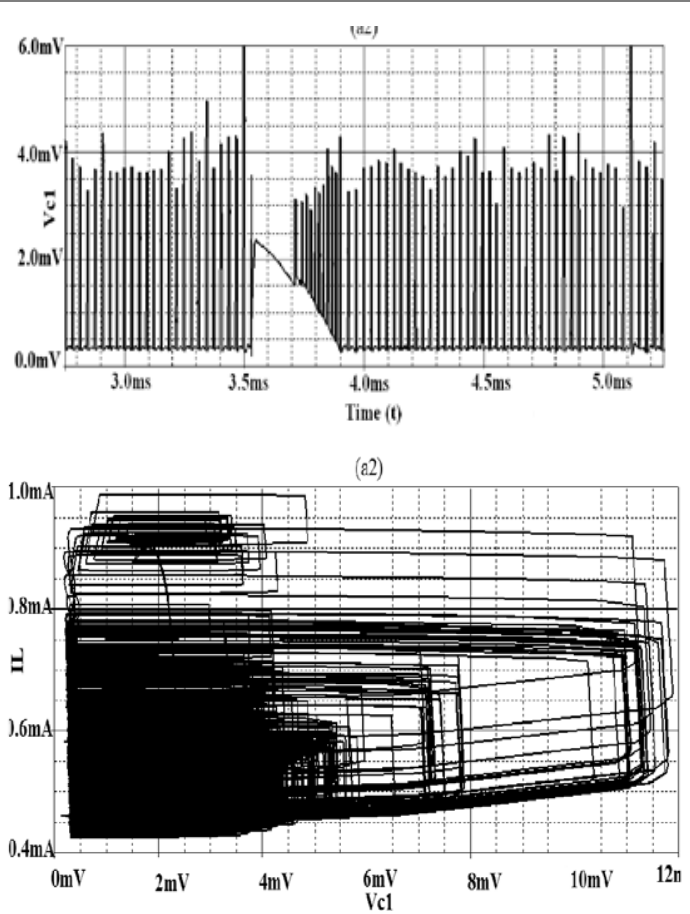

Figure 9: Results of the Pspice simulations, obtained for $R_{3}=7 \mathrm{k} \Omega$ and for the LM675 model of operational amplifier. The values of the others parameters are given in $\mathrm{Eq}(11)$. (a2): Time dependent signal voltage $v_{1}(\mathrm{t})(\mathrm{a} 2)$ : Phase space plot. Showing the chaotic pulse signals generated by the system, provided that the behaviour of the system is sensitive to the choice of operational amplifier. parameter as $\sigma$ outlined in Section 2. Secondly for two different choices of the operational amplifiers, that is to show that the dynamics of the system is also sensitive to the nature of operational amplifiers. And the obtained results are given as follows:

When the LM759CP model of the operational amplifier is used, as shown in Figure 7, and for different choice of $\mathrm{R}_{3}$, it appears that the dynamics of the system is not more rich and the system exhibits only regular periodic pulse-like behavior, but $\mathrm{R}_{3}$ must be chosen in intervals $4.68 \mathrm{k} \Omega \leq R_{3} \leq 15.6 \mathrm{k} \Omega$ and, $19.5 \mathrm{k} \Omega \leq R_{3} \leq 50 \mathrm{k} \Omega$ agreeing with results of numerical simulations. That is the ability of the system to generate regular pulses signals.

When the LM675 model of operational amplifier is used, as depicted in Figures 8-11, the dynamics of the system is more complex and the system exhibits a regular pulse like behavior (Figure 8), chaotic pulse like behavior (Figures 9 and 10) and transient chaotic pulse like behavior (Figure 11).

\section{Concluding Remarks}

In this paper, we have presented an autonomous chaotic pulse oscillator, using two stages operational amplifier coupled by mean of diode employed as the nonlinear device element, as well as a new mathematical model for a better description of its nonlinear dynamics. Using the bifurcation diagram, the dynamics of the system has been characterized with respect to the transfer voltage gain of operational amplifiers. It was found that this system exhibits regular pulse and

(bl)

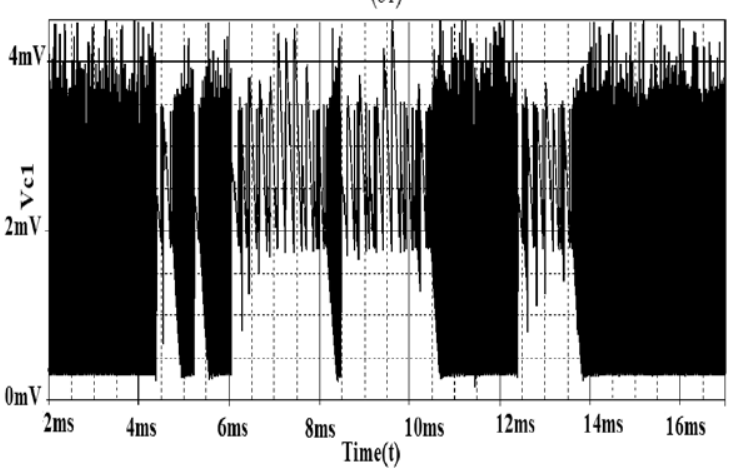

(a2)

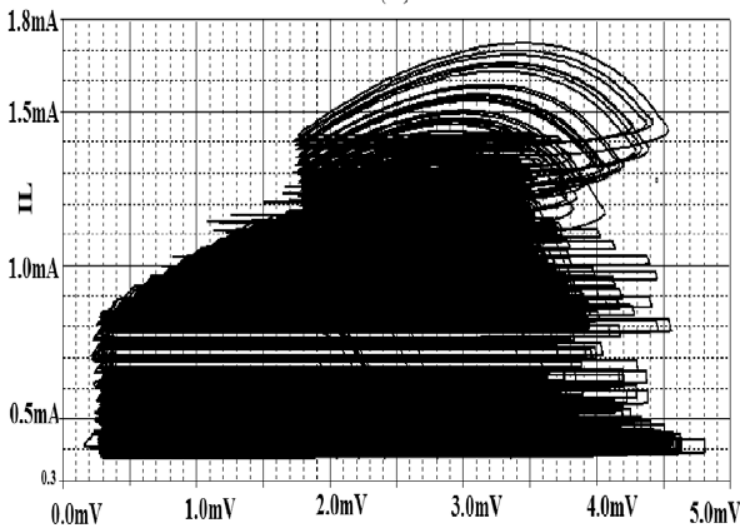

Figure 10: Results of the Pspice simulations, obtained for $R_{3}=10 \mathrm{k} \Omega$ and for the LM675 model of operational amplifier. The values of the others parameters are given in $\mathrm{Eq}(11)$. (b1) Time dependent signal voltage $\mathrm{v}_{1}(\mathrm{t}),(\mathrm{a} 2)$ Phase space plot showing the chaotic behavior of the system. 
Citation: Ndombou GB, Marquié P, Fomethe A, Yemélé D, Jeutho MG, et al. (2016) Chaotic Pulse Generation Induced by a Specific Class of Autonomous Oscillator. J Electr Electron Syst 5: 181. doi:10.4172/2332-0796.1000181

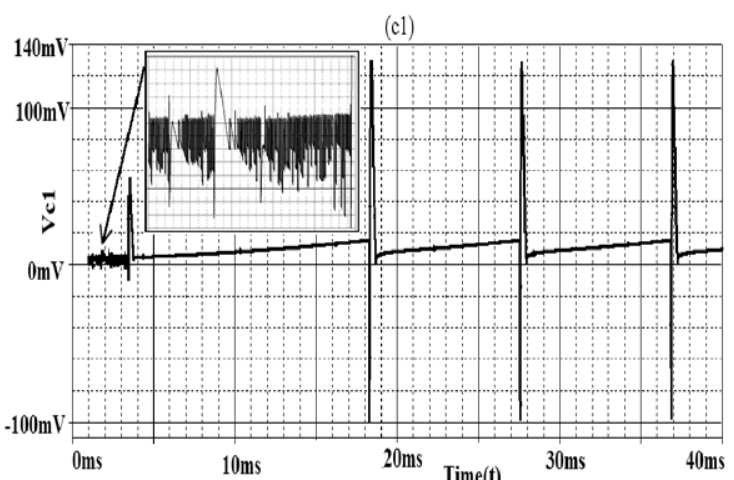

(c2)

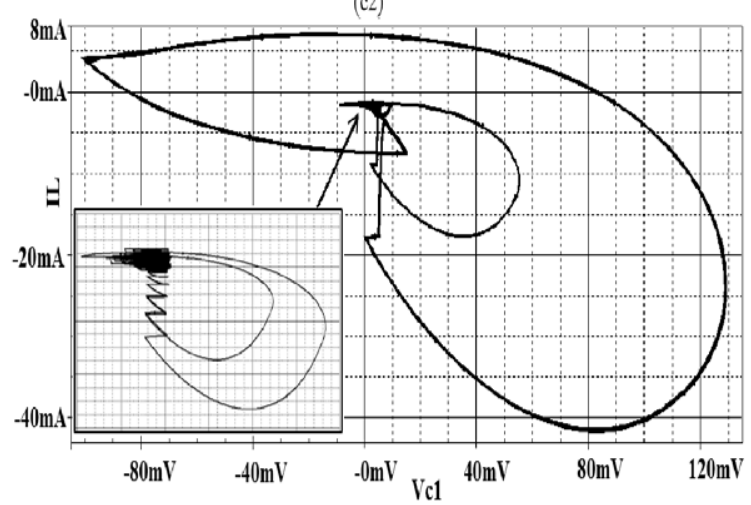

Figure 11: Results of the Pspice simulations, obtained for $R_{3}=40 \mathrm{k} \Omega$ and for the LM675 model of operational amplifier. The values of the others parameters are given in Eq (11). (C1) Time dependent signal voltage $\mathrm{v}_{1}(\mathrm{t})$ (C2) Phase space plot. As one can see, the system exhibits transient chaotic pulse like behavior.

chaotic pulse oscillations for certain range of the control parameters that are the negative resistance and the transfer voltage gain. Justifying then the dependency of the dynamics of the system on the nature of operational amplifiers. These obvious results were agreed by Pspice investigations, which confirm the dependency of oscillations on the nature of operational amplifiers and which for certain classes of operational amplifiers show new behavior, like transient chaotic pulse, not predicted by our analytical investigations, but which can be attributed to the layout of the components of operational amplifiers, which are not considered analytically. In future work, we will furthermore explore the influence of the layout of operational amplifier components, establish the sufficient conditions for the occurring of pulse and chaotic pulse signal, and try to find the exact pulse solution of the obtained dynamical equations.

\section{References}

1. Bhowmick SK (2014) How to generate chaotic pulse. International Journal of Nonlinear Science 17: 67-70.

2. Francis CM, Lau, Chi K, Tse (2003) Chaos-based digital communication systems: operating principles, analysismethods, and performance evaluation. Springer.

3. Argyris A, Syvridis D, Larger L, Lodi VA, Colet P, et al. (2005). Chaos-based communications at high bit rate using commercial fiber-optic links. Nature 438 : 343-346.

4. Rulkov NF, Sushchik MM, Tsimring LS, Volkovskii AR (2001) Digital Communication Using Chaotic-Pulse-Position Modulation. IEEE Transactions On Circuits And Systems-I: Fundamental Theory And Applications 48: 12.

5. Kengne J, Kenmogne $F$ (2014) On the modeling and nonlinear dynamics of autonomous Silva-Young typechaotic oscillators with flat power spectrum. Chaos.

6. Poliashenko M, McKay SR, Smith CW (1991) Hysteresis of synchronoussynchronous regimes in a system of two coupled oscillators. Physical Review A 43: 5638-5641.

7. Kwuimy CAK, Woafo $P$ (2008) Dynamics, chaos and synchronization of selfsustained electromechanical systems with clamped-free flexible arm. Springer 53: 201-213.

8. Torikai H, Saito T, Schwarz V A (1997).Chaotic Pulse Generator and Sawtooth Control for Information processing. IEEE International Symposium on Circuits and Systems 1: 9-12.

9. Dmitriev AS, Kyarginsky, Ye B, Panas Al, Starkov SO (2003) Experiments on ultra wideband direct chaotic information transmission in microwave band. Int J. Bifurcation and Chaos 13: 1495-1507

10. Dmitriev A, Efremova E, Kuzmin L, Atanov N (2007) Forming pulses in nonautonomous chaoticoscillator. Int. J. Biffurcation and Chaos 17: 3443-3448.

11. Giannakopoulos K, Deliyannis K (2009) Autonomous 4-D hyperchaotic oscillator and synchronization. IEEE Press Piscataway.

12. Deliyannis T, Sun Y, Fidler JK (1999) Continuous-Time Active Filter Design. CRC Press LLC.

13. Hanias MP, Giannaris G, Spyridakis A, Rigas A (2006) Time series analysis in chaotic diode resonator circuit. Chaos, Solitons and Fractals 27: 569-573. 